\title{
PEGIDA: Rechtspopulistischer Protest als Schlïsselthema zur Erschließung jugendlicher Mentalitäten?
}

\author{
Katharina Trittel · Julian Schenke
}

Eingegangen: 19. Dezember 2019 / Überarbeitet: 11. Mai 2020 / Angenommen: 7. Oktober 2020 / Online publiziert: 23. Oktober 2020

(C) Der/die Autor(en) 2020

Zusammenfassung Eignet sich das zwischen 2014 und 2017 besonders virulente Protestphänomen „PEGIDA“, um die Strukturen (post-)adoleszenter politischer Mentalitäten in einem sich verändernden politischen Klima zu erschließen? Dass es dafür aussagekräftige Anhaltspunkte gibt, soll hier in der Rückschau auf ein Forschungsprojekt diskutiert und in Bezug zu den seitherigen gesellschaftspolitischen Entwicklungen gesetzt werden.

Schlüsselwörter Protest · Rechtspopulismus · Politische Kultur · Jugend

\section{PEGIDA: Right-wing populist protest as a key issue for developing youthful mentalities}

\begin{abstract}
Between 2014 and 2017, the right-wing populist movement "PEGIDA" has been one of the most controversial, if not divisive short-term political topics in Germany. But did the political polarization in regard to this phenomenon influence the political awareness of German adolescents and young adults in a changing political climate? In this article, we suggest that there are indeed conclusive signs as revealed in our retrospective to a research project and discussion of some more recent sociopolitical developments.
\end{abstract}

Keywords Civic culture $\cdot$ Protest $\cdot$ Right-wing populism $\cdot$ Youth

\footnotetext{
Dr. K. Trittel · Dr. J. Schenke $(\bowtie)$

Institut für Demokratieforschung, Georg-August-Universität Göttingen, Weender

Landstraße 14, 37073 Göttingen, Deutschland

E-Mail: julian.schenke@demokratie-goettingen.de

Dr. K. Trittel

E-Mail: katharina.trittel@demokratie-goettingen.de
} 


\section{Erkenntnisinteresse des Forschungsprojekts ${ }^{1}$}

Die PEGIDA-Proteste ab Winter 2014/15 sorgten, ausgehend von Dresden, bundesweit für Aufsehen. Insbesondere in den folgenden Jahren suchten Wissenschaft, Medien und Politik nach Orientierungsrastern zur Einordnung des neuartigen rechtspopulistischen Protestbündnisses. Bis in den Winter 2016/17 hinein wurden die Protestmotive sowie das sozialstrukturelle und weltanschauliche Profil der PEGIDADemonstranten intensiv beforscht (vgl. Vorländer 2016, S. $53 \mathrm{ff}$.). In diesem Kontext entstand auch eine qualitative Fokusgruppenstudie zu den Politik- und Demokratievorstellungen von PEGIDA-Demonstranten (vgl. Geiges et al. 2015). Begleitet und schließlich abgelöst wurden diese explorativen Erhebungen von Überlegungen, inwieweit die Dresdner Proteste und ihre verschiedenen bundesweiten Ableger die Formierung neurechter Netzwerke innerhalb der Zivilgesellschaft beflügelt haben mögen bzw. welche Ursachen (vgl. Fehser 2017; Geiges et al. 2015, S. 179 ff.; Reuband 2017; Steinhaus et al. 2017) und Konsequenzen (vgl. Rehberg 2016; Wagner 2017; Weiß 2017) sie angesichts der unmittelbar anschließenden AfD-Erfolge für die politische Kultur der Bundesrepublik zeitigen könnten. Ist PEGIDA Symptom oder Katalysator von Strukturveränderungen der politischen Kultur der Bundesrepublik? Diese häufig angeführte und spürbar von der Sorge um zerrinnende gesellschaftliche Integrationskräfte getragene Frage galt es ab 2016 zu untersuchen.

Auch wir erweiterten den Blick auf das potenzielle gesamtgesellschaftliche Resonanzfeld der PEGIDA-Deutungsofferten, ausgehend von unterschiedlichen Orten des Protests. Als Gegenstand unserer Studie wählten wir eine Gruppe, die aufgrund ihres Lebensalters und der damit verbundenen relativen Offenheit - wenn nicht Volatilität - ihrer politischen Bewusstseinsbildung häufig als Seismograf gesamtgesellschaftlicher Veränderungen identifiziert wird: die Jugend. Nicht von ungefähr wurden Dynamiken unversöhnlicher identitätspolitischer Polarisierung, wie sie bereits seit Längerem das politische Klima der Vereinigten Staaten prägen (vgl. Hochschild 2016; Lütjen 2016), dort gerade unter zukunftsskeptischen und vom soziokulturellen Abstieg bedrohten Jugendlichen ausgemacht (vgl. Putnam 2016; Vance 2017), denen die Verheißungen des traditionellen „American Dream“ (Erfolg durch Leistung) kaum noch eine plausible Erfahrungsgrundlage bieten. Trifft die Rede von einer vergleichbaren politischen Spaltung der gesellschaftlichen Mitte im Falle eines mehr und mehr post-volksparteilichen Deutschlands zu (vgl. Münkler und Münkler 2019), so müssten zumindest Spuren einer politischen Polarisierung (vgl. Reckwitz 2017) auch in den Reihen des hiesigen Nachwuchses zu finden sein. Anzeichen dafür konnte man bereits im Kontext der NoPegida-Demonstrationen ausmachen: Hier wurde, wie eine Fokusgruppenstudie mit NoPegida-Aktivisten 2016 zeigte, ein Ensemble hochgebildeter, eher weltoffener Teile der bürgerlichen Mitte unter Einschluss von

\footnotetext{
1 Dieser Artikel basiert auf Ergebnissen eines von April 2016 bis Februar 2018 vom Bundesministerium für Familie, Frauen, Senioren und Jugend geförderten Forschungsprojekts am Göttinger Institut für Demokratieforschung unter dem Titel: „Die Jugend im Bann von PEGIDA? Zur Erforschung einer neuen zivilgesellschaftlichen Gefahr für die Demokratie“. Mitarbeiter und Ko-Autoren waren neben den beiden Verfassern dieses Artikels Christopher Schmitz und Stine Marg, denen hier nachdrücklich zu danken ist. Die aus dem Projektzusammenhang entstandene Monographie (Schenke et al. 2018) ist Ergebnis unserer gemeinsamen Arbeit.
} 
Jugendlichen und jungen Erwachsenen davon herausgefordert, den Kampf um die Präsenz auf der Straße und damit auch um die politisch-kulturelle Deutungshoheit aufzunehmen (vgl. Marg et al. 2016).

Im Zuge unseres an vorherige Studien anschließenden Forschungsinteresses galt es, die Debatten der Jugendforschung ins Feld aktualitätsbezogener politikwissenschaftlicher und politiksoziologischer Fragestellungen zu überführen. Unser Vorhaben war es, gemäß eines qualitativ-empirischen Verständnisses der politischen Kulturforschung nach Karl Rohe an die ,ungeschriebenen Verfassungen“ (Rohe 1994, S. 2) und handlungsleitenden sozialen Deutungsmuster (vgl. Oevermann 2001) kurz: die politischen Mentalitäten - von Jugendlichen und jungen Erwachsenen zu gelangen. Nicht die Herausstellung von Regelmäßigkeiten in den Einstellungsmustern war also unser Ziel, sondern - gemäß den allgemeinen Gütekriterien qualitativer Sozialforschung (vgl. Mayring (1999, S. 14) sowie Strübing et al. (2018)) - die Suche nach Sinnstrukturen, die die politische Kultur des sozialen Verbandes „Jugend“ umreißen, indem sie ,so etwas wie einen mit Sinnbezügen gefüllten politischen Denk-, Handlungs- und Diskursrahmen“ bereitstellen, ,innerhalb dessen sich das Denken, Handeln und öffentliche Reden politischer Akteure vollzieht" (Rohe 1994, S. 1).

Das leitende Forschungsinteresse der übergeordneten Studie, in dessen Rahmen auch das diesem Beitrag zugrundeliegende Material erhoben wurde, lautete somit: Hat es das Protestphänomen PEGIDA vermocht, durch eine diskursive Verschärfung der Auseinandersetzung zwischen Befürwortern und Gegnern einer migrationsgesellschaftlichen Zukunft der Bundesrepublik eine politische Polarisierung auch unter Jugendlichen und jungen Erwachsenen zu evozieren? Lassen sich im Rahmen einer qualitativ-explorativen Studie spezifische „PEGIDA-Effekte“ im Hinblick auf diese Gruppe ausmachen? Auf dieser Grundlage soll eingedenk der hier erforderlichen Engführung diskutiert werden, inwiefern, erstens, das konkrete Protestphänomen PEGIDA bei Jugendlichen und jungen Erwachsenen in den Jahren 2016 und 2017 Anklang fand und ob, zweitens, die als wesentliche inhaltliche Versatzstücke PEGIDAs identifizierten Themenfelder Eingang in die ,ungeschriebene Verfassung“ der Jugendlichen und jungen Erwachsenen im Hinblick auf Demokratie, Politik und das gesellschaftliche Zusammenleben finden.

\section{Konzeptuelle Rahmung: Diskussion um die Einordnung von PEGIDA in das Themenfeld eines ,neuen Cleavage“}

Der Dresdner Protest verband die Agitation gegen Zuwanderung im Allgemeinen und Zuwanderung durch Muslime im Speziellen früh mit einer Rhetorik des fundamentalen Misstrauens gegenüber Regierenden, politischen Institutionen, Parteien und Medien im Namen eines von ihm selbst deklamierten Volkswillens, reicherte sie mit einem besonderen sächsischen Lokalpatriotismus an, der auf die friedliche Revolution von 1989 zurückgeführt wurde und heftete all diese Motive an islam- und migrationsfeindliche, antifeministisch wie autoritär durchsetzte und immer wieder auch latent völkische Politikvorstellungen (vgl. Geiges et al. 2015, S. 89 ff.). Kurzum: PEGIDA verhalf einem Ensemble von nationalprotektionistischen bis ethnozen- 
tristischen Inhalten zu Aufmerksamkeit und Geltung. Die „Patriotischen Europäer gegen die Islamisierung des Abendlands“" organisierten und integrierten vielgestaltigen, an unterschiedlichen Einzelthemen entzündlichen Unmut zu einem Protestbündnis im Geiste jener rechtspopulistischen Formationen, wie sie seit einigen Jahren in Gestalt von Parteien, Bewegungen und Volkstribunen weltweit Erfolge feiern. Galt Deutschland lange Zeit als Fels in der Brandung einer europäischen Welle rechtspopulistischer Wahlsiege (vgl. Cuperus 2015), so lässt sich PEGIDA nun rückblickend als Auftakt einer bundesrepublikanischen politisch-kulturellen Eruption auffassen, die schließlich in den Aufstieg der ab 2015 nationalkonservativ dominierten AfD mündete.

Aus diesem Grund liegt es nahe, den Auftritt von PEGIDA als Indiz für die Genese eines neuen „Transnationalismus“-Cleavage (vgl. Hooghe und Marks 2018) zu werten, welcher quer zu den traditionellen volksparteilichen Konfliktlinien verläuft und zur immer schärferen Konfrontation zwischen eher hochgebildeten, eher mobilen Verfechtern einer weiteren Transnationalisierung von Politik und Gesellschaft, der nationalstaatlichen Kompetenzdiffusion und der Freizügigkeit von Kapitalströmen und Personen einerseits und stärker lokal verwurzelten, eher gewerblich orientierten und von Abstiegssorgen geplagten Befürwortern einer restriktiven Ordnungspolitik mitsamt sozialpolitischer Bevorzugung der autochthonen Bevölkerung andererseits verläuft (vgl. Cuperus 2015, S. 155 ff.; Geiges 2016, S. $138 \mathrm{f}$.). Zwischen diesen Konfliktfronten (ob sie sich entlang der hier verhandelten Themenkonstellationen verstetigen oder nicht, dazu vgl. Hooghe und Marks 2018, S. 127) scheint sich ein grundsätzlicher und tendenziell verbitterter Streit über die Frage abzuzeichnen, wer überhaupt legitimiert ist, sich als repräsentationsbedürftigen und repräsentierbaren Teil des „Volks“ zu definieren (vgl. Manow 2019). Sicherlich wird diese Auseinandersetzung immer wieder auch in stark simplifizierter Weise im politischen Streit geführt, was zu wissenschaftlicher Zurückhaltung nötigt. Unser Vorhaben, die ,ungeschriebene Verfassung“ der politischen Kultur von Jugendlichen und jungen Erwachsenen in den konzeptuellen Rahmen der Cleavage-Perspektive einzufassen, ist mithin als Versuch zu verstehen, die herausgearbeiteten Sinnstrukturen in die gegenwärtigen gesamtgesellschaftlichen Strukturveränderungen mentalitätsbildender Konfliktlinien (und damit der politischen Kultur) einzuordnen (vgl. Schenke et al. 2020).

\section{Methodische Konzeptualisierung}

Das periodisch wiederkehrende öffentliche Interesse an der Jugend als politischem Subjekt oszilliert zwischen der Suche nach einem „Hoffnungsträger im Zukunftsloch“ (Roth und Rucht 2000, S. 9) einerseits und Bedenklichkeiten über eine möglicherweise ,unpolitische“ bzw. entpolitisierte Jugend andererseits. Schon die Zeit der Jugendbewegung im frühen 20. Jahrhundert prägte ein bis heute wiederkehrendes Muster: Sie stellte ein politisch-kulturelles Reservoir an Verheißungen zukunftsweisender gesellschaftsverändernder Impulse vonseiten der Jugend bereit, wie es jüngst wieder durch den Auftritt der „Fridays for Future“-Bewegung reaktiviert werden konnte; und sie präformierte ein komplementäres Unwohlsein beim Ausbleiben der- 
artiger Impulse, eine ebenso zyklisch auftretende Diagnose ,verlorener“ Generationseinheiten (vgl. Koebner et al. 1985) und ,politikverdrossener“ jugendlicher Mentalitäten (vgl. etwa Pickel 2002). Abseits dieses eigenartig pathetisch anmutenden Erwartungsdualismus aber ergibt es selbstverständlich Sinn, aus den Orientierungen, Werthaltungen und Deutungsmustern von jungen Erwachsenen Rückschlüsse auf die künftige Integrität von Gesellschaft und Demokratie - salopp: auf ihr Wohl und Wehe - zu ziehen. Was sie heute beschäftigt und bewegt, mag auf die zentralen gesellschaftlichen Themen und Konfliktfelder von morgen hindeuten (vgl. Hoffmann-Lange 2006, S. 55; Schäfers und Scherr 2005, S. 21), auch wenn die tatsächliche Prägekraft aktueller (post-)adoleszenter Stimmungen und Überzeugungen mit Rücksicht auf entsprechende biografische Veränderungsprozesse im Zuge eines fortschreitenden Lebensalters (vgl. Schröder 2018) sowie auf unvorhersehbare gesamtgesellschaftliche Großentwicklungen vorsichtig abwägend beurteilt werden muss.

Zudem: Wer sich in das Feld der Jugendforschung begibt, stellt schnell fest, dass sich Fragen nach dem Zustand ,der“ Jugend in zunehmendem Maße an der tatsächlichen Heterogenität sich ausdifferenzierender und zugleich expandierender adoleszenter und postadoleszenter Lebenswelten brechen (vgl. Merkens 2008). Dass auch die Altersgrenzen der Phasen von Adoleszenz und Postadoleszenz aufgrund historischer Ausdehnungsprozesse (verursacht etwa durch die Bildungsexpansionsdynamik und den Wandel der Erwerbswelt) nicht allein biologisch determiniert sind (vgl. Schäfers und Scherr 2005, S. 23), ist seit der zweiten Hälfte des 20. Jahrhunderts wiederholt festgestellt worden. Frühere Entwicklungsphasenmodelle, die die Jugendphase als psychosoziales „Moratorium“ zwischen durch Abhängigkeit gekennzeichneter Kindheit und der Selbstständigkeit des Erwachsenenalters mitsamt den dazugehörigen Fähigkeiten zu strukturierter Erwerbsarbeit und zur Verantwortungsübernahme sowie charakterlicher Integrität verstanden, gelten daher schon seit einiger Zeit als veraltet (vgl. Vetter 2006, S. 25 ff.). Die zunehmende Diskontinuität von Erwerbsbiografien, auch der wachsende diskriminatorische Effekt sozialer Ungleichheiten und von Bildungs- sowie Statusdifferenzen zwischen den Herkunftsfamilien (vgl. Merkens 2008) verstärken diese Erosion eines einst linear konzipierten Modells von „Jugend“, was auch an den mitunter stark variierenden Altersspannen unterschiedlicher Jugendstudien abzulesen ist (vgl. Schenke et al. 2018, S. 27). Gerade politische Urteilsbildung formiert sich häufig noch bis über das 30. Lebensjahr hinaus (vgl. Hoffmann-Lange 2006, S. 59; Pickel 2002, S. 26). Eine Studie, die auf das gesellschaftspolitische Denken und Empfinden ,der“ Jugend abzielt, sollte daher ein möglichst breites empirisches Sample abbilden.

Aus diesen Überlegungen ergibt sich das Erfordernis einer projektbezogenen Konzeptualisierung des Untersuchungsgegenstands „Jugend“. Nicht gesucht wurde etwa nach entwicklungsphasenspezifischen Sozialisationsfaktoren, d.h. nach den Bedingungen des Erlernens oder Erwerbs von politischen Wissensgehalten und Urteilsvermögen. ,Jugend“ bezeichnet im vorliegenden Projektzusammenhang junge Erwachsene zwischen 16 und 35 Jahren, verstanden als heterogene soziale Gruppierung mit untereinander vergleichbaren Lebenslagen, Erfahrungsräumen und Präferenzmustern. Rekrutierungsbedingt umfasste unser Sample zu zwei Dritteln Personen im Alter zwischen 19 und 30 Jahren. 
Da die allgemeine Entwicklung jugendlicher Orientierungen durch die Shell Jugendstudien, den Längsschnittsurvey des Deutschen Jugendinstituts und durch die SINUS-Milieustudien bereits materialreich dokumentiert wird (vgl. Schenke et al. 2018, S. $26 \mathrm{ff}$.), entschieden wir uns für eine qualitativ-empirische Studie mit thematischem Fokus auf das Auftreten und die Themen von PEGIDA. Unser Vorgehen orientierte sich dabei an den von Strübing et al. jüngst zusammengestellten Gütekriterien qualitativer Sozialforschung, insbesondere an der Forderung nach Gegenstandsangemessenheit, empirischer Sättigung und Originalität (vgl. Strübing et al. 2018, S. 85, 88 und 94). In der Tradition politischer Kulturforschung nach Karl Rohe stehend, untersuchten wir einerseits die diskursiv verhandelte politische ,Deutungskultur" von Jugendlichen und jungen Erwachsenen, suchten andererseits aber auch nach den tieferliegenden Sinnstrukturen ihrer politischen ,Soziokultur“, also nach Anhaltspunkten ,unbewusster“, d.h. handlungsleitender politischer Weltbilder und latenter Selbstverständlichkeiten, die das Gesagte sinnhaft vermitteln (vgl. Rohe 1990) und im Rahmen einer ,ungeschriebenen Verfassung“ Handlungsorientierungen an die Hand geben (Rohe 1994, S. 2). Die Methode der Wahl, um derartige Deutungsmuster aufzudecken, war die Gruppendiskussion bzw. die hier weitgehend eingesetzte Fokusgruppendiskussion. Die Fokusgruppe, bisweilen auch als ad-hocGruppe bezeichnet, ist eine spezifische Variante der Gruppendiskussion, die nach bestimmten Kriterien zusammengesetzt wird und in welcher die Teilnehmer über ausgewählte (eben ,fokussierte“) Themen diskutieren. Im Gegensatz zur Realgruppe kennen sich die Fokusgruppenteilnehmer untereinander nicht (vgl. Kühn und Koschel 2011, S. 20 und 77). Die Fokusgruppe zielt darauf ab, den Aushandlungsprozess alltäglicher Interaktionen - und damit auch die Modi und Inhalte politischer Urteilsbildung in Bezug auf spezifische Themen - in beobachtbarem Rahmen abzubilden, ohne auf das Korsett standardisierter Antwortmöglichkeiten zurückgreifen zu müssen (vgl. Kühn und Koschel 2011, S. 105; Marg 2014, S. 72). Sie simuliert den stets interaktiv gestifteten Prozess politischer Urteilsbildung auf wirklichkeitsnahe Weise, indem sie den Teilnehmern ermöglicht, ihre persönlichen Relevanzsysteme und Semantiken abseits vorab festgelegter Antwortoptionen zu entfalten. Die hier ausgewerteten Gruppendiskussionen (darunter 9 Fokusgruppen und 3 Realgruppen) wurden durch einen Themenkatalog strukturiert, welcher den inhaltlichen Fokus sowie ein gewisses Maß an Vergleichbarkeit zwischen den Diskussionsrunden sicherstellte. Zwei Moderatoren leiteten die Gespräche, die aufgezeichnet und anschließend verschriftlicht wurden. Die hieraus entstandenen Transkripte wurden mithilfe der Software MAXQDA anschließend deduktiv (Aspekte, die sich vorab aus der Forschungsfrage ergaben) sowie induktiv (Aspekte, die erst in der Analyse der Gesprächsverlaufe entdeckt wurden) codiert. So gelangten wir zu „Schlüsselkategorien“ (Przyborski und Wohlrab-Sahr 2010, S. 211) der sozialen Deutungsmuster der Jugendlichen und jungen Erwachsenen. Auf Grundlage der Codierungen fertigten wir fallimmanente wie fallübergreifende Thesenpapiere sowie argumentierende Interpretationen an, die zusammen mit den Notizen der bei den Diskussionen ebenfalls anwesenden Protokollanten im Forscherteam diskutiert und anschließend zu interpretierenden Auswertungskapiteln verdichtet wurden.

Da nach einem potenziellen Einfluss von PEGIDA auf die jungen Erwachsenen gesucht wurde, fanden die Fokusgruppendiskussionen in Städten statt, die als Mo- 
bilisierungszentren des Protestbündnisses auffielen. Wir begannen mit einer ersten Erhebungswelle in Dresden und Leipzig im Sommer 2016, d. h. in jenen Städten, die wir infolge unserer kontinuierlichen Forschungsarbeit zu den lokalen Bedingungen und zum Wandel von PEGIDA nicht nur als entscheidende Demonstrationsorte von PEGIDA und NoPegida identifizieren konnten, sondern auch als Städte, in denen die diskursive Polarisierung zwischen Sympathisanten und Gegnern das lokalkulturelle Klima zumindest vorübergehend prägte. Zunächst rekrutierten wir hier per E-MailVerteiler junge Erwachsene aus Sportvereinen, Jugendeinrichtungen und ähnlichen Institutionen. Da sich in diesen ersten vier Diskussionen jedoch eine starke Übereinstimmung der Positionierungen mit jenen der bereits 2016 beforschten (zu großen Teilen ebenfalls aus Jugendlichen und jungen Erwachsenen bestehenden) NoPegidaAktivisten herauskristallisierte, beauftragten wir Markt- und Meinungsforschungsstudios mit der Rekrutierung von Diskussionsteilnehmern für weitere Fokusgruppen nach spezifischen Parametern (Geschlecht paritätisch, niedrige wie hohe Bildungskarrieren, größere altersmäßige Streuung), um einen hinsichtlich Bildungsniveau und politischer Haltung ausgewogeneren Realitätsausschnitt zu erzielen. Zudem versuchten wir, mithilfe eines weiteren Parameters die Fokusgruppen hinsichtlich der weltanschaulich-politischen Orientierungen maximalkontrastiv gegenüberzustellen. Salopp: Migrationskritische versus migrationsfreundliche, „rechte“ versus „linke“ Fokusgruppen. Wir ließen potenzielle Teilnehmer zur Positionierung zu einer bewusst suggestiv gewählten These auffordern: „Ich habe Angst vor einer Ausbreitung des Islam in Deutschland und Europa“. Daraufhin sortierten wir die Teilnehmer vor$\mathrm{ab}$ in die heuristischen Teilgruppen der „Beunruhigten“ (Angst bis große Angst) und der „Unbekümmerten“ (wenig bis keine Angst), um mit ihnen jeweils zwei weitere Diskussionen durchzuführen. Die Annahme lautete, dass „Angst vor einer Ausbreitung des Islam in Deutschland“ als euphemisierende rhetorische Hülse dienen kann, um migrationskritische und migrationsfeindliche Positionen zu legitimieren. $\mathrm{Zu}$ Vergleichszwecken folgte eine zweite Erhebungswelle im Frühling und Frühsommer 2017 mit vier Diskussionen in Nürnberg und Duisburg, zwei westdeutschen Orten mit aktiven PEGIDA-Ablegern, mit gleichbleibenden Rekrutierungsparametern. Tatsächlich funktionierte die Kontrastierung wie erwartet: Unter den „Beunruhigten“ waren migrationskritische und migrationsfeindliche Auffassungen mehrheitsfähig, während die „Unbekümmerten“ mehrheitlich eine kosmopolitische (wenn auch, wie weiter unten dargelegt, durch abwertende Äußerungen über Migranten durchzogene) Gruppenmoral etablierten. Als Materialgrundlage unserer Auswertung dienten insgesamt zwölf Fokusgruppen mit einer Dauer von je 120 bis 150 min, an denen 88 Personen teilnahmen. Ergänzt wurden die daraus entstandenen rund 720 Transkriptseiten durch einen Fragebogen, der soziodemografische Daten sowie das Wahlverhalten der Teilnehmer erfasste. 


\section{Ergebnisse}

\subsection{Primäre Distanz zur Protestformation PEGIDA}

Die Untersuchung diskursiver Auswirkungen einer Protestbewegung, die zumindest anfangs heterogene Anliegen artikulierte und unterschiedliche Gruppen anzog, somit als „Spiegel“, „Projektionsfläche“ oder auch „leerer Signifikant“ (Heim 2017) bezeichnet werden konnte, ist diffizil.

Sicher, es gibt identifizierbare Themenkomplexe von PEGIDA, wie etwa die mit Verve zelebrierte Politikerschelte als Bestandteil einer populistischen Mobilmachung gegen ,die Elite“ (vgl. zu dieser Strategie Müller (2016) und Priester (2019)). An eine solche Rhetorik schließt sich indes einer von drei Aspekten an, die PEGIDA - so unsere aus der Feldforschung und Rezeption der Forschungsliteratur extrapolierte Ausgangsannahme - als mögliche Anschlussstellen für junge Menschen offeriert: Die Stoßrichtung gegen die von Akteuren des rechten Spektrums proklamierte (vermeintliche) Hegemonie (links-)liberaler Eliten (vgl. dazu auch Biess 2019; Koppetsch 2019; innerhalb der „neuen Rechten“ vgl. Sellner 2017), welche die öffentliche Meinung und politische Stimmungslage beeinflussen und das Dogma der „,multikulturellen Gesellschaft“ setzen würden. Zweitens stellt sich PEGIDA gegen eine vermeintliche ,Islamisierung“ (die eine Verteidigung der „Heimat“ erforderlich mache) und das bedeutet in erster Linie: „Flucht, Asyl und Einwanderung Begriffe, die faktisch bedeutungsgleich verwendet, und damit beliebig austauschbar werden -, sind eine Bedrohung für die ,deutsche Identität", den Sozialstaat und Wirtschaftsstandort Deutschland“" (Schenke et al. 2018, S. 103; vgl. zur ökonomischen Perspektive und zum Kampf um Etabliertenvorrechte Nachtwey 2016); ein Konnex, der, drittens, auf eine von PEGIDA explizit adressierte Kosten-Nutzen-Rechnung, die als „ökonomisches Prisma“ bezeichnet werden kann, hinausläuft, in der u.a. auch Geflüchtete unter dem Aspekt der Leistungsbereitschaft beurteilt werden.

Hinsichtlich der Frage nach der Anschlussfähigkeit von PEGIDA für junge Menschen müssen zwei Bezugsebenen unterschieden werden: Einerseits das konkrete Protestereignis, die Bewegung auf der Straße, und andererseits PEGIDA als allgemeiner diskursiver Platzhalter für die soeben genannten Themen der politischen Rechten. Diese Zweiteilung spannt den Rahmen für den Kernbefund unserer Studie: Die von uns untersuchten Gruppen halten eine primäre Distanz zu PEGIDA als konkretem Protestphänomen, zeigen aber gleichwohl eine sekundäre (und damit ist gemeint: diskursive) Anschlussfähigkeit an bestimmte Themen. Im vorliegenden Beitrag soll eruiert werden, ob diese Anschlussfähigkeit der jungen Menschen an PEGIDA (oder vice versa) - also: PEGIDA-Effekte - in den genannten identifizierbaren Offerten PEGIDAs liegt oder (auch) in impliziten politisch-sozialen Deutungsmustern der jungen Menschen.

Mit Blick auf die erste Bezugsebene kann ein klares Urteil gefällt werden: Die von uns Befragten zeigten sich en gros nicht als Anhänger der „Patriotischen Europäer“. Niemand bekannte sich dazu, schon einmal an den Spaziergängen teilgenommen zu haben, es fanden sich keine rhetorischen Adaptionen der von PEGIDA geprägten Begriffe - kurzum: ihre Rituale, Symboliken und Selbstvergewisserungspraktiken verfangen bei den jungen Menschen nicht und hatten in unserer Studie keine Be- 
deutung über den bewegungsinternen Horizont hinaus. Die von PEGIDA als Teil eines Labels (vgl. Marg und Trittel 2016) aufgebaute kollektive Identität ist für sie wenig reizvoll. Themen, die Menschen motivierten, bei PEGIDA teilzunehmen (Heimatverbundenheit; das vermeintlich berechtigte inhaltliche Anliegen; das Gefühl, mit dem Protest eine Sprachlosigkeit überwinden zu können; das Gefühl, ,die Politik“ lasse einen im Stich, diffamiere sogar den Protest; ferner ein ausgeprägtes Misstrauen gegenüber den Medien (vgl. Geiges et al., S. $97 \mathrm{ff}$. )), entfalteten in unserem Sample keine Strahlkraft.

Diese klare Distanz rührt jedoch nicht primär von den Themen her, die PEGIDA vertritt, sondern vielmehr aus der Haltung der Mehrzahl unserer Diskutanten (unabhängig von Rekrutierungshintergrund, Wertorientierungen oder sozioökonomischen Faktoren), die Protest im öffentlichen Raum (insbesondere Demonstrationen) als potenziell illegitime Störung öffentlicher Abläufe beurteilen. Die jungen Menschen zeigen sich also kaum protestaffin und stehen damit im eklatanten Widerspruch zu NoPegida-Aktivisten ${ }^{2}$, die den öffentlichen Raum nachgerade als ebenjenen Ort ansehen, an dem Demokratie praktisch generiert und auch verteidigt werde, ,erst auf der Straße werde man zum Bürger“ (Marg et al. 2016, S. 113). Da die Straße umkämpft sei, weisen NoPegida-Aktivisten die von den jungen Menschen geforderte Reglementierung dieses Raumes dezidiert zurück (Marg et al. 2016, S. 114).

Die jungen Menschen in unseren Gesprächsrunden unterstellen, Protestierende würden die öffentliche Bühne primär für Störaktionen bis hin zur Gewalt nutzen (woraus sie die repressive Forderung ableiten, legitimer Protest habe dort stattzufinden, wo er die Mehrheit der Gesellschaft nicht störe) und dadurch das reibungslose Funktionieren des öffentlichen Lebens (insbesondere des Nahverkehrs) beeinträchtigen. Durch die erforderlichen Sicherheitsmaßnahmen würden zudem ,Steuergelder verschwendet“. Diejenigen, welche die Protestmotive nachvollziehen können und die artikulierten Forderungen grundsätzlich goutieren, verweisen allerdings eher resigniert darauf, dass Protest kein wirkungsvolles Mittel sei, um gesellschaftliche Veränderungen erreichen zu können.

Auf der Suche nach „PEGIDA-Effekten“ muss zusätzlich bedacht werden, dass die Teilnehmenden unserer Studie von sich aus das Protestphänomen nicht thematisiert haben. Nichtsdestotrotz stellt sich die Frage, inwiefern PEGIDA in sekundärer Weise als diskursiver Platzhalter, als Projektionsfläche und Vehikel bestimmter Deutungsmuster fungiert. Gehen wir davon aus, dass PEGIDA zu einer „Profanisierung des rechtspopulistischen Diskurses“ (vgl. Institut für Demokratieforschung 2016) beigetragen und somit Grenzen der Sagbarkeit verschoben hat, ergibt sich die Frage, inwiefern die von PEGIDA vorgetragenen Themen in der vermeintlichen bürgerlichen „Mitte der Gesellschaft“ (vgl. Reckwitz 2020) Resonanz erzeugen, also die bereits erwähnte ,sekundäre Anschlussfähigkeit“ bei den jungen Menschen gegeben ist.

\footnotetext{
2 In der qualitativen Studie zu NoPegida waren rund 55\% der insgesamt 54 Teilnehmenden der Fokusgruppen zwischen 16 und 35 Jahre alt (vgl. Marg et al. 2016, S. 21), während die ca. 30 Teilnehmenden der Fokusgruppen mit Pegida-Demonstrierenden alle Altersstufen zwischen 18 und ca. 65 Jahren abdeckten (vgl. Geiges et al. 2015, S. 91).
} 


\subsection{Sekundäre Anschlussfähigkeit über ausgewählte Topoi}

\subsubsection{Relevanzsystem und politisch-soziale Deutungsmuster}

In unserer Studie kristallisierten sich jenseits von den genannten Rekrutierungsparametern und sozioökonomischen Unterschieden acht Gruppen heraus, denen die Teilnehmenden zugeordnet wurden. Die Gruppierung erfolgte über die Auswertung der Codings der individuellen Gesprächsbeiträge, wobei vor allem (kritisch reflektierend) berücksichtigt wurde, über welche Attribute sich die Teilnehmenden selbst beschrieben haben. ${ }^{3}$ Es handelt sich hierbei um keine Typenbildung im streng sozialwissenschaftlichen Sinne, sondern vielmehr um die Beschreibung einer Bündelung von Auffälligkeiten.

Zunächst lassen sich die jungen Menschen des gesamten Samples bezüglich ihres Relevanzsystems und der Strukturierung ihres Werteraumes im Sinne eines mehrdimensionalen Konstruktes (vgl. Lechleiter 2016, S. 36) als überwiegend konventionalistisch, konformistisch und kompatibilitätsorientiert beschreiben. Da aufgrund der geringen Teilnehmerzahl der einzelnen Gruppen sinnvolle Differenzierungen innerhalb des Samples nicht immer möglich sind und - so viel sei vorweggenommen - sich die Gruppen bezüglich der Frage nach „PEGIDA-Effekten“ kaum signifikant unterscheiden, sollen sie ergänzend mit den in früheren Studien befragten Teilnehmern der PEGIDA- bzw. der NoPegida-Proteste in Beziehung gesetzt werden.

Die dominanteste Gruppe (1: aktive Realisten) besteht vor allem aus Studierenden, ist überwiegend an Stabilität orientiert, hält traditionelle Lebenskonzepte, in deren Zentrum die Familie und ,der Traum vom Spießer-Eigenheim“ stehen, für erstrebenswert, konsumiert politische Inhalte in zurückhaltend-bekömmlicher Weise und zeigt ein Bewusstsein für das Allgemeinwohl, welches sie aber eher im persönlichen Nahbereich (Familie, Freunde) und weniger gesamtgesellschaftlich verortet. Die Dominanz des klassischen Familienideals zeigt sich noch ausgeprägter bei denjenigen (2: stabilitätsorientierte Materialisten), die im Sinne einer favorisierten Sesshaftigkeit explizit wieder aus der Stadt auf das Land ziehen möchten, in die Nähe ihrer Herkunftsfamilie und ihres Heimatortes, wo sie sich auf eine (bescheidene) Besitzstandswahrung fokussieren. Die Jüngsten unter ihnen (3: jugendliche Materialisten) orientieren sich darüber hinaus verstärkt an (familiären) Autoritäten, leben oftmals noch zuhause und suchen Stabilität im persönlichen Nahraum. Auch die Zugehörigen einer männlich dominierten Gruppe (4: hedonistische Materialisten) schätzen das Leben in gewohnten Bahnen: Sie möchten eine „ordentliche Beziehung“ führen, am Wochenende feiern gehen; sie definieren sich über Fitness, Gaming und Freunde. Vor allem aber sprechen sie Geld einen hohen Stellenwert zu, doch geht es nicht darum, durch ökonomische Potenz etwa Macht und Status zu erlangen, sondern dar-

\footnotetext{
3 Zu Beginn jeder Fokusgruppe wurden die Teilnehmenden aufgefordert, sich aus 64 Bildern, die als Plakate im Raum verteilt waren, drei bis maximal fünf Bilder auszusuchen, die ,Dinge zeigen, die für sie wichtig sind“. Die Bilder visualisierten sowohl ein Repertoire an Jugend- und Alltagskultur als auch politische Themen sowie Pegida-Bezüge. Ein freies Feld konnte zudem als ,Joker“ gefüllt werden, falls sich Teilnehmende nicht in der Auswahl wiederfinden konnten. Bei der Vorstellung der von ihnen ausgewählten Bilder wurde der Gruppe erläutert, was konkret mit den Abbildungen assoziiert wurde, um möglichst früh im Gespräch alltagsweltliche Erfahrungen und Handlungsparadigmen einfangen zu können.
} 
um, sich abzusichern - ,genug zu haben“, damit „alles ein bisschen schöner“ sei. Gewissermaßen potenziert wird die Einstellung der stabilitätsorientierten Materialisten von einer Gruppe (5: ordnungsliebende Materialisten), die überwiegend als Beunruhigte rekrutiert wurden, stark an Stabilität interessiert und an Materialismus, Sicherheit und Schutz ausgerichtet sind. Die sentimental durchsetzte Idealisierung einer überschaubaren mittelstandsgesellschaftlichen Vergangenheit ist hier Tenor, ein bedauerndes „Ist ja heute nicht mehr so wie damals“ eine typische Floskel. Entsprechend prangern sie heutige Missstände an und wenden sich explizit von bestimmten Werten wie Vielfalt ab. Gemeinschaft und Heimat sind für sie ebenso wie für die PEGIDA-Teilnehmer, für die Heimatverbundenheit einen expliziten Antrieb zu protestieren darstellte (Geiges et al. 2015, S. 97), besonders wichtig, doch exkludieren sie bestimmte Gruppen dezidiert aus diesem Konzept. Auf der Suche nach Übersichtlichkeit beklagt eine kleine Gruppe (6: realistische Zweifler) den Zustand einer Welt, in der man sich nur schwer zurechtfände, allerdings ohne eine Schuldzuweisung in Richtung Politik zu adressieren. Für sie steht die Bewältigung ihres Alltags mit seinen Widrigkeiten im Zentrum, sie sind auf der Suche nach Zugehörigkeit.

Auf dem eher kosmopolitischen Pol der Werteskala lässt sich eine Gruppe (7: nonkonforme Idealisten) verorten, deren Angehörende meist als Unbekümmerte rekrutiert wurden und formal hoch gebildet sind, und die sich im Gegensatz zu den in den anderen Gruppen dominanten Beharrungskräften mehr an den Fliehkräften, an Veränderung und Selbstverwirklichung orientieren. Sie meiden die Klischees des Eigenheims oder der traditionellen Familie, definieren ihr Leben eher fluide: „Ich kann mir das überhaupt nicht vorstellen, längere Jahre in einem Land zu leben, und ich habe auch gar nicht so das Bedürfnis, wirklich jetzt irgendwie eine Familie zu gründen oder dann 40 Jahre mit einem Partner zusammenzuleben [...] es ist wirklich so, dass ich mir das eher viel mehr so vorstelle, dass ich wirklich meine Entscheidungen nicht an, irgendwie, einen großen menschlichen Anhang, dann, binden muss, sondern einfach wirklich dorthin ziehen kann, wo ich hinziehen möchte, je nachdem, wie es mir gerade geht". Als einzige Gruppe messen sie Politik und gesellschaftlicher Veränderung durch Meinungsäußerung einen großen Stellenwert bei, wobei sie explizit positive Veränderungen durch Zuwanderung thematisieren. Materielles ist dem Ideellen untergeordnet. Eine letzte Gruppe (8: Fast-Sozialökologische) oszilliert zwischen den bisher beschriebenen Motiven: Zwar sind sie an Stabilität interessiert, doch wird diese eher an Wertvorstellungen festgemacht, die sich auf Rücksichtnahme, auch Umweltschutz, beziehen. Interessanterweise wird dieser auch als Lebensraumschutz, z. T. auch im Sinne eines Heimatschutzes, interpretiert. Die Rücksicht, welche man der Natur als Lebensgrundlage entgegenbringt, gilt allerdings nicht unbedingt bspw. für Geflüchtete. Prosoziale Werte wie Menschlichkeit, Toleranz und Vielfalt sind hier Schlagworte - manchmal allerdings auch, wie noch diskutiert wird, nicht mehr.

Betrachtet man diese Gruppencharakteristika, ergeben sich weitere mögliche Anschlussstellen an die Themen von PEGIDA: das Leitbild der traditionellen Familie, die ambivalente Verhandlung der Werte Vielfalt und Toleranz, der hohe Stellenwert von Recht und Ordnung als Ausdruck von Konformität und dem Bedürfnis nach Sicherheit (in der Abwehr von „Fremdem“), die Frage nach kollektiver Zugehörigkeit zu einer bestimmten Gruppe im Kontrast zu einem Konzept gesellschaftlicher 
Solidarität, allgemeiner gefasst: die Frage nach dem Politik- und Demokratieverständnis der jungen Menschen. Denn die bereits einleitend aufgeworfene Frage, ob sich neue polarisierungsfähige Konfliktlinien durch die Gesellschaft ziehen (vgl. Reckwitz 2017), verweist auf das politische Potenzial der jungen Menschen (zum Zusammenhang der Polarisierungsfrage und ihrer Auswirkungen auf die politische Bildung von Jugendlichen, vgl. Milbradt et al. 2018).

Die Wahlpräferenzen der von uns Befragten waren verglichen mit dem bundesrepublikanischen Trend wenig überraschend: Die CDU verliert Zuspruch, während die Grünen und die AfD Stimmenanteile gewinnen konnten und der Anteil der Nichtwähler leicht rückläufig ist. Zum Vergleich: Die NoPegida-Aktivisten gehörten überwiegend dem linken politischen Lager an (vgl. Marg et al. 2016, S. 103) und wählten 2015 überwiegend die Grünen und die Linkspartei; Nichtwähler waren in diesem Kreis deutlich unterrepräsentiert. Wer 2016 bei PEGIDA mitlief, gab überproportional oft der AfD seine Stimme (vgl. Institut für Demokratieforschung 2016, S. 20). Doch umfasst das politische Potenzial natürlich mehr als den Urnengang; die Beteiligung am System, so das Selbstverständnis der NoPegida- und PEGIDAAktivisten, sichere dessen Fortbestand. Ganz anders verhält es sich indes mit den jungen Menschen aus unserem Sample: Politik spielt (mit Ausnahme der nonkonformen Idealisten) und z. T. im Gegensatz zu den Befunden der 18. Shell Jugendstudie (vgl. Schneekloth und Albert 2019) eine klar untergeordnete Rolle. Da jedoch eine vordergründig distanzierte Haltung nicht als umfassende apolitische Einstellung missverstanden werden sollte, haben wir uns dafür entschieden, die politischen Gehalte der Diskussionsrunden unter den Begriff des ,unsichtbaren Politikprogramms“ (Begriff geprägt durch Kohl und Seibring 2012) zu subsumieren. Denn obwohl die jungen Menschen erwartbare Vokabeln meiden, sprechen sie dennoch über virulente gesellschaftspolitische Themen und präsentieren sich als politisch Urteilende, insofern, dass sie darüber nachdenken, was für das eigene Leben und die persönliche Rolle in der Gesellschaft wichtig und richtig ist.

Es fällt auf, dass die jungen Menschen das, was in Politik und Gesellschaft funktioniert, als gegeben annehmen. Eine funktionsfähige Gesellschaft ist entsprechend dieser Deutung nicht das Resultat gesteuerter Prozesse und Aktionen, gar einer - wie bei NoPegida artikuliert wurde - aktiven Streitkultur (Marg et al. 2016, S. 108), sondern gewissermaßen überzeitlich vorhanden. Diese Vorstellung speist sich offenbar auch aus einer grundsätzlichen Zufriedenheit mit dem Funktionieren des Systems und nicht aus einer etwa bei PEGIDA vorherrschenden Frustrations- oder Enttäuschungserfahrung, welche dort allerdings in politische Aktion und die Suche nach alternativen Lösungen transferiert wurde (vgl. Geiges et al. 2015, S. 114 und S. 116). Im Gegensatz zu den aufbegehrenden PEGIDA-Aktivisten engagieren sich unsere Diskutanten, dem Umkehrschluss folgend, dass man bei mittlerer Funktionsfähigkeit nicht aktiv werden müsse, nicht. Für sie scheint die Diagnose der NoPegida-Aktivisten, ,die jahrelange Herrschaft der Merkelregierung habe sich wie Mehltau über das Land gelegt", was die Bevölkerung einschläfere und ihr politisches Desinteresse erkläre (Marg et al. 2016, S. 108), zu gelten.

Politik wird für die jungen Menschen vielmehr erst dann relevant, wenn etwas mit konkretem Bezug zur eigenen Alltagsrealität nicht zu funktionieren scheint. In solchen Fällen werden offenkundig Ängste oder Sorgen geweckt. Obwohl zwei Drittel 
der Befragten nicht explizit über Parteien, Politiker oder politische Inhalte sprachen (hier taten sich im Vergleich die Diskutanten von PEGIDA und NoPegida viel stärker hervor, vgl. Marg et al. 2016, S. 198), erscheinen die jungen Menschen nicht als politikverdrossen oder politisch apathisch (vgl. Gille 2018). Vielmehr nehmen sie Politik in vier Dimensionen wahr: (1) als Gefühl, (2) als Dezision, (3) als Synonym für den Staat und (4) als zu konsumierendes Produkt. In der heutigen Misstrauensgesellschaft zeigen, erstens, Sentenzen, die mit ,,ich glaube“ oder ,es fühlt sich so an" beginnen, dass man der Politik nicht vollständig traut, dass die Macht der Stimmungen einen großen Einfluss auf die eigene Wahrnehmung hat. Das Vertrauen in die Politik würde sich verstärken, wenn, zweitens, mehr entschieden würde; deutlich teilen die jungen Menschen die Sehnsucht der PEGIDA-Teilnehmer nach Letztentscheidungen, die die Bewältigung zentraler Probleme ermöglichen würden. Es geht um die Entscheidung an sich, nicht um ihre Ausverhandlung und den Prozess der Entscheidungsfindung. Da staatliche Institutionen (wie Arbeitsamt oder Schulbehörde) drittens mit Politik gleichgesetzt werden, diese aber gleichwohl als unverrückbar gelten, entbinden sich die jungen Menschen mit dieser Deutung von der Notwendigkeit, sich zu beteiligen. Sie stehen hiermit in einem spürbaren Kontrast zu dem in der aktuellen Shell Jugendstudie formulierten Untertitel „Eine Generation meldet sich zu Wort" (Albert et al. 2019a), der darauf abzielt, dass die Jugend nun verstärkt eigene gesellschaftliche Gestaltungsansprüche anmelden würde. In unserem Sample präsentieren sich die jungen Menschen vielmehr, viertens, als Konsumbürger im Sinne einer konsumierten Politik (vgl. auch Lamla und Neckel 2006, S. 7), die wie ein Selbstbedienungsladen erscheint (dieses Bild wählt auch Walter 2018, S. 10): Man sucht sich heraus, was einem gefällt und gerade benötigt wird, bevorzugt Produkte, ,die einem auf Augenhöhe präsentiert werden, nach denen man sich weder bücken noch strecken muss“ (Schenke et al. 2018, S. 207). Weder möchte man die Regale einräumen, noch das Sortiment mitbestimmen. Mit dieser Haltung stehen sie im eklatanten Widerspruch zu den Aktivisten von NoPegida, die gegenteilig postulieren, dass Demokratie eben nicht bereit-, sondern immer wieder aufs Neue hergestellt und mitunter auch erkämpft werden müsse (Marg et al. 2016, S. 112). In ihren Augen beginne die Demokratie sich sogar aufzulösen, sobald die Bürger sich in den privaten Raum zurückzögen. Aufgrund dieses Befundes sprechen wir bezüglich unseres Samples von Politikdistanz bzw. von politischer Unberührtheit, die der eigentliche Grund der beobachteten Polarisierungsresistenz der Jugendlichen und jungen Erwachsenen zu sein scheint.

Eine ähnlich pragmatisch-konsumorientierte Haltung legen die jungen Menschen in Bezug auf das stets sehr heterogen gefüllte Konzept „Demokratie“ an den Tag. Sie unterscheiden sich hinsichtlich ihres Demokratieverständnisses von den Aktivisten von PEGIDA und NoPegida in vier Punkten: (1) Die Kenntnis demokratischer Praktiken, (2) das Recht, gehört zu werden, (3) Partizipation und (4) die Einsicht in die Prozesshaftigkeit von Demokratie.

PEGIDA-Aktivsten zeigen sich zwar unzufrieden mit der Politik, aber dennoch politikinteressiert. Sie stehen nicht für den ,,apathisch-desinteressierten Nichtwähler" (Geiges et al. 2015, S. 107), sondern sind im Gegensatz zu den jungen Menschen bezüglich ihrer Erwartungen an Politik sprechfähig. Auch mit Demokratie kennen sie sich besser aus als die politisch nicht aktive gesellschaftliche Mitte. Sie 
rücken den Aspekt der Volksherrschaft ins Zentrum, und übersetzen Demokratie mit „Wille des Volkes“. Sie fordern eine ,gesellschaftliche Diskussionskultur“, die sie allerdings durch ,Sprechverbote“ beschnitten sehen (Geiges et al. 2015, S. 108) eine Deutung, die auch auf eine bewusste Selbststigmatisierung hindeutet. Gleichwohl erkennen sie aber an, dass Demokratie ein langwieriger Prozess sei, dass zur demokratischen Diskussion der Widerstreit von Meinungen und das Finden von Kompromissen gehöre (Geiges et al. 2015, S. 107).

Für die jungen Menschen bedeutet Demokratie in erster Linie, dass man als Teil des Volkes bestimmte Rechte besitzt. Allerdings beziehen sie sich nicht wie PEGIDA auf einen exkludierenden Volksbegriff; dieser bleibt eher diffus im Sinne einer Mehrheitsmeinung, von der sie annehmen, sie zu vertreten. Denn auffällig ist: Die jungen Menschen setzen ihre Bedürfnisse mit dem Allgemeinwohl gleich, auch weil sie offenbar keine Vorstellung von der Legitimität haben, eigene gruppenspezifische politische Interessen zu identifizieren und auf demokratischem Wege durchzusetzen. Vielmehr dominiert eine „Es ist, wie es ist“-Haltung, die den Wunsch, politisch etwas verändern zu wollen, vermissen lässt. Sie sehen sich mehr als Teil einer Gemeinschaft denn als (aktiven) Teil der (abstrakt und fern bleibenden) Gesellschaft; diese Selbstverortung teilen sie mit den PEGIDA-Aktivisten, die sich selbst als ,das Volk" (verstanden als organische, homogene Einheit) und Gemeinschaft imaginieren, die sie einer atomisierten Gesellschaft entgegen stellen (Geiges et al. 2015, S. 111). Der von PEGIDA betonte prozedurale Charakter von Demokratie ist im Bewertungshorizont der jungen Menschen überhaupt nicht, dafür umso mehr bei NoPegida-Aktiven präsent: Diese denken Demokratie als reversiblen, dynamischen und langfristigen Aushandlungsprozess (Marg et al. 2016, S. 111), in dem technokratische Zwänge und Parteieninteressen in den Hintergrund zu treten haben und insbesondere Minderheitenrechte zu schützen seien. Während die jungen Menschen sich kaum mit demokratischen Praktiken auskennen, fordern die NoPegida-Aktivisten eine Bürgerpflicht ein, sich an der Demokratie zu beteiligen. Durch intensive gemeinsame Diskussion könne sie alsdann ein emanzipatives Potenzial entwickeln, „das nicht auf die Sicherung des Bestehenden fokussiert ist, sondern die gemeinsame Weiterentwicklung der Gesellschaft im Blick hat“ (Marg et al. 2016) - eine Perspektive, die in unserem Sample maximal die Gruppe der nonkonformen Idealisten einnehmen würde. Vor diesem Hintergrund ist es auch nicht verwunderlich, dass sowohl die bei PEGIDA als auch bei NoPegida im Protest Engagierten Widerspruch als positiv für eine Demokratie bewerten, weil er Fortschritt bedeute; entgegen des Postulats der jungen Menschen, dass das Fehlen von Reibungen, das Funktionieren, der ideale gesellschaftliche Zustand sei, wobei sie auch die Realisierung der Demokratie anders als PEGIDA als überwiegend positiv bewerten.

\subsubsection{Das ökonomische Prisma und das rissige Dogma der multikulturellen Gesellschaft}

Ein hervorstechender, alle Gruppen umschließender Befund ist die große Dominanz klassischer bürgerlicher Wertvorstellungen unter den jungen Menschen, die sich insbesondere in dem meritokratischen Ideal eines leistungs- und bildungsbasierten sozialen Aufstiegs manifestieren. Es herrscht fast durchgehend die Vorstellung, dass 
Freiräume, Erfolg und Wohlbefinden das Resultat individueller Leistungsbereitschaft sind, Bildung gilt als der entscheidende Karrieremotor. Daraus resultierend wollen sich die jungen Menschen im individualistischen Alleingang in die Reihen einer grundsätzlich meritokratisch orientierten Mitte durchkämpfen als Manager des eigenen Schicksals (vgl. auch Albert et al. 2019b, S. 43) - eine Haltung, die auf das Hier und Jetzt fokussiert, auf eine ,,eingefrorene ' ewige Gegenwart, ohne historische, soziale oder politische Entstehungs- und Erhaltungsbedingungen“" (Schenke et al. 2018, S. 244) und die weitere Facetten des schon beschriebenen unsichtbaren Politikprogramms offenbart. Die Fokussierung auf dieses Mittelschichtsideal geht über alle Bildungs-, Rekrutierungs- und Erwerbsgrenzen hinweg: Der zentrale Orientierungsrahmen ist eine deutsche Adaption des American Dream inklusive seines Aufstiegsversprechens. Mit dieser Haltung reproduzieren die jungen Menschen ohne den Begriff zu benutzen - die Mitte als „Sehnsuchtsort“ (Marg 2014, S. 33), als Stabilitätsanker, als Referenzpunkt gesellschaftlicher Normalität, aber auch als Chiffre für sozialen Aufstieg, der durch Bildung und Leistungsbereitschaft erreicht werden könne und der lange Zeit auf der plausiblen Überzeugung fußte, dass Leistung und Anstrengung in der Zukunft honoriert würden; eine Perspektive, die ein umfassendes Gerechtigkeits- und Aufstiegsversprechen barg und die Berufstätigkeit als Bedingung für Selbstständigkeit und Freiheit antizipierte. Als Grundlage dafür gilt Bildung, die einen wettbewerbsfähig mache, der allerdings auch (ähnlich wie von den NoPegida-Aktivisten) eine gesellschaftsverändernde Kraft zugeschrieben wird, sodass sie nicht selten zum (präventiven) Patentrezept gegen rechte Mobilisierungsversuche gerinnt. So besitzt Bildung Problemlösungskompetenzen nicht nur für die eigene Biografie, sondern auch für gesellschaftliche Konflikte. Und obwohl meritokratische Überzeugungen auf lange Sicht nur für die flexiblen, gebildeten, ressourcenstarken Erwerbstätigen einer Gesellschaft attraktiv sein können, da eine solche Gesellschaftsordnung soziale Ungleichheit legitimiert und potentielles Scheitern der Verantwortlichkeit des Einzelnen zuschiebt, vertreten die jungen Menschen ihre meritokratische Überzeugung trotzig. Wenn auch, so scheint es, weniger aus Überzeugung, sondern vielmehr aus einer sozialisationsbedingten Grundhaltung, die aufgrund des Fehlens übergreifender Orientierungsrahmen weiterhin wirksam bleibt.

Überspitzt ließe sich unser Sample mit dem Signum „Generation Agenda 2010“ versehen: Nicht nur, weil die Diskutanten ihre Jugend in den 2000er-Jahren verbrachten, sondern vor allem wegen dieses Leistungs- und Bildungsimpetus, ihrer arbeitgeberfreundlichen Erwerbsmoral und aufgrund des „ökonomischen Prismas“, durch das sie urteilen. Sie erkennen durchaus die soziale Grundproblematik, dass viele mit wenig auskommen müssen. Doch nehmen es die meisten als naturgegeben hin, dass die Gesellschaft in Arm und Reich zerfalle, was man nicht ändern könne, sich deshalb auch nicht solidarisch-politisch organisieren müsse, weil im Ernstfall der Sozialstaat als sorgender Vater zur Stelle sei - ein Vertrauen, dass etwas überraschend auch NoPegida-Aktivisten teilen (Marg et al. 2016, S. 116). Diese Perspektive bündelnd formuliert ein knapp 20-jähriger Studienabbrecher in kompromissloser Konsequenz: „Also ich war immer zufrieden mit meinem Leben, deswegen hat mich Politik eigentlich nie interessiert".

Gesellschaft und die Rolle von Individuen in ihr werden also durch ein ökonomisches Prisma (wer verdient aufgrund seiner eigenen Leistung was) betrachtet, 
welches gleichfalls als Deutungsfolie für Geflüchtete angelegt wird: Größtenteils ist man empathisch gegenüber denjenigen, die weniger haben, solange diese ebenfalls dem uneingeschränkt verfochtenen Leistungsparadigma anhängen würden, also einen Gewinn für die Gesellschaft bedeuteten (vgl. zum „unternehmerischen Selbst“ Bröckling 2007, S. 56). Diejenigen, insbesondere der ordnungsliebenden Materialisten, welche am ehesten den Positionen von PEGIDA zuneigen, fokussieren allerdings vor allem auf den eigenen Status, für sie gilt: „Hast Du nichts, bist Du nichts“. Diese Haltung verbindet sich oftmals mit einer populistischen Elitenfeindschaft und mit sozialchauvinistischen Kulturkampf-Motiven. Die zudem von den jungen Menschen getroffene Unterscheidung in Geflüchtete, die wirklich vor Not und Krieg flöhen, und solchen, die als „Wirtschaftsflüchtlinge“ ins Land kämen, ist bereits ein bekanntes Muster nicht nur aus den Gesprächsrunden mit PEGIDATeilnehmern (Geiges et al. 2015, S. 122), wobei hier nicht Migranten, sondern ein mit Terror und Gewaltbereitschaft assoziierter „Islam“ das eigentliche Problem darstellte (Geiges et al. 2015, S. 123), sondern wurde bereits als fester Bestandteil rassistischer Diskurse in Deutschland nachgewiesen (vgl. Hövermann et al. (2015) und Jäger (1992, S. 217 ff.)). Wie antizipiert, ist also die Beurteilung von „Fremden“ durch dieses Prisma, gekoppelt mit den Themen Sicherheit und Kultur, eine subkutane Anschlussstelle an die Themen von PEGIDA. Allerdings ist diese Perspektive gleichfalls bei NoPegida-Aktivisten und PEGIDA-Teilnehmern erkennbar, was den Befund relativiert: Von Gruppen, zu denen man sich selbst nicht zählt, wird uni sono gefordert, dass sie zunächst eine bestimmte Lebensleistung vorweisen und einen Beitrag zur Gesellschaft leisten müssten, bevor sie Ansprüche anmelden oder sich denunzierend gegen andere wenden dürften (vgl. Geiges et al. 2015, S. 127).

Das Zusammenleben in der multikulturellen Gesellschaft ist also alles andere als friktionsfrei, sondern voller Hürden und Ambivalenzen. Bereits Marg hat das „Bezugsproblem multikulturelle Gesellschaft“" als gemeinsamen Deutungsrahmen innerhalb des von uns untersuchten Samples ausgemacht und darauf hingewiesen, dass dieser eigenständig von den jungen Menschen in den Diskussionsrunden angesteuert wurde - anders als das Thema PEGIDA (vgl. Marg 2019). Das Sprachmuster ist fast immer identisch: Zunächst wird der Wert einer multikulturellen Gesellschaft, die von Vielfalt lebe und Toleranz hervorbringe, betont, und erst im weiteren Verlauf darauf hingewiesen, ja mitunter bitter beklagt, dass sie eben auch (ein Zuviel an) Toleranz einfordere, die Thematisierung ihrer Schattenseite jedoch einer gesellschaftlichen Tabuisierung unterliege. Gerade die NoPegida-Aktivisten stellen ihre praktizierte Toleranz demonstrativ PEGIDA und der Mehrheitsgesellschaft entgegen, sodass Toleranz als „eine Art Masterframe der NoPegida-Proteste“ gedeutet worden ist (Marg et al. 2016, S. 140). Nichtsdestotrotz zeigte sich auch in dieser Gruppe, dass Toleranz auch die Vorstufe zur Indifferenz bedeuten kann, keinen stabilisierenden Wert, sondern eher einen Minimalkonsens des Zusammenlebens definiert, der den jungen Menschen jedoch offenbar keinen Halt zu geben im Stande ist. Das ,gesellschaftliche Toleranzgebot" scheint so eher dazu angetan, das Fehlen eigener Werte zu verdecken. Die multikulturelle Gesellschaft erscheint für die jungen Menschen somit zwar als tief inkorporiertes, ,normatives Leitbild“ (Marg 2019, S. 119), das sie jedoch auf Grundlage von Alltagserfahrungen - wenn auch erst im zweiten Schritt - in Frage stellen. Sie sind damit aufgewachsen, dass es zu dem Bekenntnis zum 
„Multikulti-Land“ keine gesellschaftlich goutierten Alternativen gibt. Doch erleben sie in ihrem Alltag Probleme im Zusammenhang mit kultureller Heterogenität, die zu diesem Credo in Kontrast stehen. Während die NoPegida-Aktivisten fordern, dass auch Geflüchtete die Straße als öffentlichen Raum für sich beanspruchen sollten (Marg et al. 2016, S. 114), äußern die jungen Menschen gerade hierüber so manche Sorge, erzählen von ,,am helllichten Tage glotzenden“ Männergruppen aus dem ,arabischen Raum“. Doch fehlt das Vokabular, die Sorgen zu benennen, ohne sich dem Verdacht auszusetzen, das Dogma der Toleranz anzuzweifeln, als Rassist etikettiert zu werden. Pointiert gesprochen: Sobald die jungen Menschen über alltägliche Begebenheiten sprechen, wird deutlich, dass sie von der praktizierten Toleranz im Umgang mit oder der Beurteilung von etwa Geflüchteten weit entfernt sind; das Postulat der Toleranz erscheint als gesellschaftlich auferlegte, aber leere Monstranz, wie der Wortbeitrag einer Teilnehmerin verdeutlicht: „Multikulti, das kann ja nicht funktionieren [...] Und multikulti, das ist so abgelutscht, das Wort. Also ich kann es nicht mehr hören! Das ist, ja, das muss man cool finden. Das muss man toll finden. Der Witz ist bloß: Ich muss als Deutsche alles toll finden. Die finden uns aber nicht toll. Ja?“. Es zeigt sich: Anders als im Urteil von Calmbach et al., das positive Bild einer pluralen Zivilgesellschaft sei bei Jugendlichen „,noch nicht gefestigt und selbstverständlich präsent" (Calmbach et al. 2016, S. 427), zeigen unsere Befunde eine enorme Präsenz, jedoch gleichfalls mitnichten eine gefestigte Inkorporierung aus Überzeugung. Die Diskussion um Vielfalt und Toleranz offenbart, dass diese Werte für die Teilnehmenden nur schwer zu füllen sind und kaum als handlungsleitend interpretiert werden können. Sie wissen, dass sie sich irgendwie dazu verhalten müssen, flüchten sich aber gerade deshalb in vage Aussagen oder äußern, wie die erwähnte Teilnehmerin, dezidierte Ablehnung. Drastischer formuliert: Die anfänglichen Lippenbekenntnisse zur multikulturellen Gesellschaft dienen letztlich als Disclaimer für die straflose Sagbarkeit rassistisch eingefärbter Deutungsmuster, die sich in erster Linie auf eine stereotype Abwertung des „Fremden“ beziehen und nahezu unabhängig von Rekrutierung, Wahlpräferenz oder Gruppencharakteristika vorgenommen werden (Häufungen finden sich lediglich bei den ordnungsliebenden Materialisten). Damit zeigt sich eine größere Verteilung auf die Gruppen des Samples als in der Shell Jugendstudie von 2019, in der lediglich die PopulismusGeneigten (24\%) und die Nationalpopulisten (9\%) durch Ressentiments auffallen (vgl. Albert et al. 2019a, S. 17).

Die von den jungen Menschen getätigten und von Marg unter dem Begriff ,,abwertende Ungleichwertigkeitsaussagen“ (Marg 2019) subsumierten Äußerungen lassen entsprechend Vorurteile, z. T. auch verfestigte Ressentiments, im Einzelfall sogar manifeste rassistische Einstellungsmuster erkennen. Dies deckt sich mit dem Befund, dass populistische Argumentationsmuster sich als anschlussfähig erweisen (Milbradt 2018, S. 216), weil sie „ein offenbar weit verbreitetes Gefühl ab[decken], dass es Dinge gibt, die man nicht ansprechen darf, ohne dafür nach subjektiver Wahrnehmung moralisch sanktioniert zu werden“ (Albert et al. 2019a, S. 16). Und tatsächlich traten auffällig häufig und oftmals unangefochten fremdenfeindliche Stereotypisierungen in den Gruppendiskussionen auf und wurden gerade von jenen geäußert, die sich zunächst zu den Prinzipien von Vielfalt, Weltoffenheit und Toleranz bekannt hatten, alsdann jedoch - das anfängliche Bekenntnis hinter sich lassend - hefti- 
ge Verurteilungen bestimmter Gruppen, zumeist Geflüchteter aus dem arabischen Raum, artikulierten.

Dieser Diskussionsmechanismus wurde in den Gruppen, die im Osten durchgeführt wurden, unmittelbarer ausgelöst als im Westen; die ,,abwertenden Ungleichwertigkeitsaussagen“" wurden allerdings - auch das muss konstatiert werden - in anderen Begrifflichkeiten und deutlich weniger kompromisslos vorgetragen, als dies bei PEGIDA der Fall ist. Indem die eigene Fremdenfeindlichkeit zurückgewiesen wird und man sie nicht ins Bewusstsein vordringen lässt, verfolgen die Diskutanten eine Externalisierungsstrategie, wie sie in Bezug auf Rechtsradikalismus auch bereits von Oevermann (vgl. Oevermann 1998; ausgeführt an aktuellen Beispielen vgl. Finkbeiner et al. 2019) beschrieben worden ist.

Obwohl die jungen Menschen überwiegend optimistisch in die eigene Zukunft blicken (Unsicherheiten, ob man den eigenen Lebensweg realisieren könne, werden äußerst defensiv formuliert, widersprechen sie doch der meritokratischen Grundeinstellung), wurden auch Ängste und Unsicherheiten thematisiert, insbesondere Abstiegsängste bezüglich der eigenen ökonomischen Sicherheit, vor (gewalttätigen) „Fremden“ im öffentlichen Raum, vor Verlust des gesellschaftlichen Zusammenhalts verbunden mit einer diffusen Ohnmacht in Bezug auf überfordernde gesamtgesellschaftliche Problemlagen - Aspekte, die vor dem Hintergrund des eingangs aufgestellten Polarisierungsverdachtes vor allem Fragen von Zugehörigkeit berühren. Und dennoch: Die durchaus sichtbare, aber keineswegs sich dominant aufdrängende Kontingenzangst der jungen Menschen scheint kein Einfallstor rechtspopulistischer Agitation zu sein, wohl aber innerhalb der Gesprächsrunden ein (wenn auch stotternder) Motor solcher Deutungsmuster, die eine sekundäre Anschlussfähigkeit an PEGIDA offerieren, auch durch die Strategie, Angst diskurstaktisch einzusetzen, um eine stärker legitimierte Sprecherposition, aber auch ,das Vorrecht auf das Vorurteil" (Schenke et al. 2018, S. 321), zu erhalten - ein Mechanismus, der sich auch in PEGIDAs Forderung offenbart, als „,besorgte Bürger“ besonderes Gehör zu verdienen. Gleichwohl handelt es sich dabei nicht um einen neuen Mechanismus, sondern vielmehr offenbart sich ein Muster, welches sich in der Analyse von Vorurteilen als Teil rechtsradikaler Bewusstseinsformen in der bundesrepublikanischen Gesellschaft konstant zeigt (vgl. Koppetsch 2018).

\section{Diskussion und Ausblick: Sekundäre Anschlussfähigkeit an PEGIDA als Ausdruck eines jugendlichen Teilmilieus?}

In gewissem Sinne ist der hier verhandelte unmittelbare Forschungsgegenstand mit den AfD-Wahlerfolgen seit 2016 obsolet geworden: PEGIDA-Proteste erregen derzeit kein landesweites Aufsehen mehr, halten nationalprotektionistische, migrationswie EU-feindliche Deutungsofferten doch mittlerweile Einzug in die Landesparlamente wie auch in den Bundestag. Doch fasst man die Auseinandersetzung zwischen PEGIDA und NoPegida als frühe Ausprägungsform einer immer deutlicher konturierten Konfliktlinie zwischen kosmopolitischen und nationalprotektionistischen Mentalitätssträngen auf, wird deutlich, dass die wesentlichen Ergebnisse der Studie auf Problemzusammenhänge von unverminderter Aktualität hindeuten. 
Um den Kontrast zumindest illustrativ anzudeuten, bietet sich der Rekurs auf ein jüngeres jugendliches Protestphänomen an: Der Auftritt von „Fridays for Future“ vitalisierte jüngst (wieder einmal) die Vorstellung einer politisierten Jugendgeneration. Häufig wird bei einer solchen Diagnose jedoch über die Milieuspezifik dieses hauptsächlich von Schülern und Studenten getragenen Protests hinweggeschritten: Im Jahre 2019 demonstrierte in erster Linie der Nachwuchs hochgebildeter und gutsituierter Elternhäuser für das Klima (vgl. Sommer et al. 2019, S. 12f.). Im Rahmen der hier vorgestellten qualitativen Studie kamen demgegenüber vor allem jene jungen Erwachsenen zu Wort, die sich einer erwerbs- und aufstiegsorientierten Mitte zurechnen. Da viele von ihnen weder Abitur ablegten noch studierten, sondern Aus- oder Weiterbildung verfolgten, konterkarierten sie ihren großstädtischen Lebensbedingungen zum Trotz jene eher studentisch und eher bildungsbürgerlich geprägten Milieus, welche etwa auch die NoPegida-Proteste maßgeblich trugen (vgl. Marg et al. 2016). Dass auch unter (Post-)Adoleszenten die Friktionen zwischen zwei Teilmilieus - auf der einen Seite die eher mobilen, hochgebildeten, migrationsgesellschaftlich-kosmopolitisch Gestimmten, auf der anderen Seites die stärker migrationsskeptisch-nationalprotektionistisch und kleinräumlich Orientierten - zunehmend die sozialen Deutungsmuster und politischen Mentalitäten, mithin die Werthaltungen, Hoffnungen, Ängste sowie Demokratie- und Gesellschaftsvorstellungen zu bestimmen scheinen, wird besonders im Vergleich unserer Studienergebnisse mit den NoPegida-Protesten von 2014-2016 oder eben auch mit „Fridays for Future" ab 2018 deutlich.

Die am Thema PEGIDA entzündeten Auseinandersetzungen, so lässt sich schlussfolgern, werden die politische Diskussion in der Bundesrepublik aller Voraussicht nach noch jahre-, wenn nicht jahrzehntelang prägen - und dabei wohl auch auf unterschiedliche Trägerthemen aufsatteln.

Funding Open Access funding enabled and organized by Projekt DEAL.

Open Access Dieser Artikel wird unter der Creative Commons Namensnennung 4.0 International Lizenz veröffentlicht, welche die Nutzung, Vervielfältigung, Bearbeitung, Verbreitung und Wiedergabe in jeglichem Medium und Format erlaubt, sofern Sie den/die ursprünglichen Autor(en) und die Quelle ordnungsgemäß nennen, einen Link zur Creative Commons Lizenz beifügen und angeben, ob Änderungen vorgenommen wurden.

Die in diesem Artikel enthaltenen Bilder und sonstiges Drittmaterial unterliegen ebenfalls der genannten Creative Commons Lizenz, sofern sich aus der Abbildungslegende nichts anderes ergibt. Sofern das betreffende Material nicht unter der genannten Creative Commons Lizenz steht und die betreffende Handlung nicht nach gesetzlichen Vorschriften erlaubt ist, ist für die oben aufgeführten Weiterverwendungen des Materials die Einwilligung des jeweiligen Rechteinhabers einzuholen.

Weitere Details zur Lizenz entnehmen Sie bitte der Lizenzinformation auf http://creativecommons.org/ licenses/by/4.0/deed.de. 


\section{Literatur}

Albert, M., Hurrelmann, K., \& Quenzel, G. (2019a). 18. Shell Jugendstudie - Eine Generation meldet sich zu Wort. Weinheim: Beltz.

Albert, M., Hurrelmann, K., \& Quenzel, G. (2019b). Jugend 2019: Zwischen Politisierung und Polarisierung. In M. Albert, K. Hurrelmann \& G. Quenzel (Hrsg.), 18. Shell Jugendstudie - Eine Generation meldet sich zu Wort (S. 35-47). Weinheim: Beltz.

Biess, F. (2019). Republik der Angst: Eine andere Geschichte der Bundesrepublik. Hamburg: Rowohlt.

Bröckling, U. (2007). Das unternehmerische Selbst. Soziologie einer Subjektivierungsform. Frankfurt a. M.: Suhrkamp.

Calmbach, M., Borgstedt, S., Borchard, I., Thomas, P. M., \& Flaig, B. F. (2016). Wie ticken Jugendliche 2016? Lebenswelten von Jugendlichen im Alter von 14 bis 17 Jahren in Deutschland. Wiesbaden: Springer.

Cuperus, R. (2015). Wie die Volksparteien (fast) das Volk einbüßten - Warum wir den Weckruf des Populismus erhören sollten. In E. Hillebrand (Hrsg.), Rechtspopulismus in Europa. Gefahr für die Demokratie? (S. 149-158). Bonn: Dietz.

Fehser, S. (2017). Demaskierung und Kontinuitäten. Pegida als Offenlegung und Entfesselung bestehender Dispositionen. In T. Heim (Hrsg.), Pegida als Spiegel und Projektionsfläche. Wechselwirkungen und Abgrenzungen zwischen Pegida, Politik, Medien, Zivilgesellschaft und Sozialwissenschaften (S. 55-78). Wiesbaden: Springer VS.

Finkbeiner, F., Trittel, K., \& Geiges, L. (2019). Rechtsradikalismus in Niedersachsen. Akteure, Entwicklungen und lokaler Umgang. Bielefeld: transcript.

Geiges, L. (2016). Nach dem Hype. Drei Entwicklungen von PEGIDA seit dem Winter 2014/15. In K.-S. Rehberg (Hrsg.), PEGIDA. Rechtspopulismus zwischen Fremdenangst und „Wende“-Enttäuschung? Analysen im Überblick (S. 129-141). Bielefeld: transcript.

Geiges, L., Marg, S., \& Walter, F. (2015). PEGIDA. Die schmutzige Seite der Zivilgesellschaft? Bielefeld: transcript.

Gille, M. (2018). Jugend und Politik - ein schwieriges Verhältnis. DJI Impulse, 1, 16-19.

Heim, T. (2017). Pegida als leerer Signifikant, Spiegel und Projektionsfläche - eine Einleitung. In T. Heim (Hrsg.), Pegida als Spiegel und Projektionsfäche. Wechselwirkungen und Abgrenzungen zwischen Pegida, Politik, Medien, Zivilgesellschaft und Sozialwissenschaften (S. 1-33). Wiesbaden: Springer VS.

Hochschild, A. R. (2016). Strangers in their own land. Anger and mourning on the American right. New York: The New Press.

Hoffmann-Lange, U. (2006). Was kann die Jugendforschung zur politischen Kulturforschung beitragen? In E. Roller, F. Brettschneider \& J. W. van Deth (Hrsg.), Jugend und Politik: ,Voll normal! “ (S. 55-74). Wiesbaden: VS.

Hooghe, L., \& Marks, G. (2018). Cleavage theory meets Europe's crises. Lipset, Rokkan, and the transnational cleavage. Journal of European Public Policy, 1(25), 109-135.

Hövermann, A., Groß, E., \& Zick, A. (2015). „Sozialschmarotzer“ - der marktförmige Extremismus der Rechtspopulisten. In I. R. Melzer \& D. Molthagen (Hrsg.), Wut, Verachtung, Abwertung. Rechtspopulismus in Deutschland (S. 95-108). Bonn: Dietz.

Institut für Demokratieforschung (2016). Büchse der Pandora? PEGIDA im Jahr 2016 und die Profanisierung rechtspopulistischer Positionen, Göttingen. http://www.demokratie-goettingen.de/content/ uploads/2016/10/Pegida2016_G\%C3\%B6ttinger_Demokratieforschung.pdf. Zugegriffen: 29. Nov. 2019.

Jäger, S. (1992). BrandSätze. Rassismus im Alltag. Duisburg: DISS.

Koebner, T., Janz, R.-P., \& Trommler, F. (Hrsg.). (1985). „,Mit uns zieht die neue Zeit“. Der Mythos Jugend. Frankfurt a. M.: Suhrkamp.

Kohl, W., \& Seibring, A. (Hrsg.). (2012). „Unsichtbares Politikprogramm?“ Themenwelten und politisches Interesse von „bildungsfernen“ Jugendlichen. Bonn: Bundeszentrale für politische Bildung.

Koppetsch, C. (2019). Die Gesellschaft des Zorns. Rechtspopulismus im globalen Zeitalter. Bielefeld: transcript.

Koppetsch, C. (2018). Ressentiments. Soziopolis, 20.12.2018. https://soziopolis.de/beobachten/gesellschaft/ artikel/ressentiments/. Zugegriffen: 29. Apr. 2020.

Kühn, T., \& Koschel, K.-V. (2011). Gruppendiskussionen. Ein Praxis-Handbuch. Wiesbaden: Springer VS.

Lamla, J., \& Neckel, S. (2006). Vorwort der Herausgeber. In J. Lamla \& S. Neckel (Hrsg.), Politisierter Konsum - Konsumierte Politik (S. 7-8). Heidelberg: VS. 
Lechleiter, P. (2016). Wertekonstellationen im Wandel. Eine empirische Bestandsaufnahme. Wiesbaden: Springer VS.

Lütjen, T. (2016). Die Politik der Echokammer. Wisconsin und die ideologische Polarisierung der USA. Bielefeld: transcript.

Manow, P. (2019). Demokratisierung der Demokratie. Merkur, 847(73), 5-15.

Marg, S. (2014). Mitte in Deutschland. Zur Vermessung eines politischen Ortes. Bielefeld: transcript.

Marg, S. (2019). Nach Pegida - Politische Orientierungen junger Menschen. Fokusgruppen als Instrument zur Deutungsmusteranalyse. In I. N. Bögelein \& N. Vetter (Hrsg.), Der Deutungsmusteransatz. Einführungen - Erkenntnisse - Perspektiven (S. 107-127). Weinheim: Beltz Juventa.

Marg, S., Trittel, K., Schmitz, C., Kopp, J., \& Walter, F. (2016). NoPegida. Die helle Seite der Zivilgesellschaft? Bielefeld: transcript.

Marg, S., \& Trittel, K. (2016). PEGIDA: Vom „Schmuddelkind“ zum professionalisierten Protestformat, Göttingen. http://www.demokratie-goettingen.de/blog/zwei_jahre_Pegida. Zugegriffen: 29. Nov. 2019.

Mayring, P. (1999). Einführung in die qualitative Sozialforschung. Eine Anleitung zu qualitativem Denken. Weinheim: Beltz.

Merkens, H. (2008). Jugendforschung. Auf der Suche nach dem Gegenstand? In A. Ittel (Hrsg.), Jahrbuch Jugendforschung 7 (S. 349-380). Wiesbaden: Springer VS.

Milbradt, B. (2018). Über autoritäre Haltungen in ,postfaktischen' Zeiten. Opladen: Barbara Budrich.

Milbradt, B., Heinze, F., \& König, F. (2018). Politische Bildung in einer Welt des Umbruchs. DJI Impulse, 1, 4-9.

Müller, J.-W. (2016). Was ist Populismus? Ein Essay. Berlin: Suhrkamp.

Münkler, H., \& Münkler, M. (2019). Abschied vom Abstieg. Eine Agenda für Deutschland. Hamburg: Rowohlt.

Nachtwey, O. (2016). Die Abstiegsgesellschaft : Über das Aufbegehren in der regressiven Moderne. Berlin: Suhrkamp.

Oevermann, U. (1998). Zur soziologischen Erklärung und öffentlichen Interpretation von Phänomenen der Gewalt und des Rechtsextremismus bei Jugendlichen. Zugleich eine Analyse des kulturnationalen Syndroms. In H.-D. König (Hrsg.), Sozialpsychologie des Rechtsextremismus (S. 83-126). Frankfurt a. M.: Suhrkamp.

Oevermann, U. (2001). Zur Analyse der Struktur von sozialen Deutungsmustern. Sozialer Sinn, 1, 3-33.

Pickel, G. (2002). Jugend und Politikverdrossenheit. Zwei politische Kulturen im Deutschland nach der Vereinigung? Wiesbaden: Springer.

Priester, K. (2019). Die Alternative für Deutschland. Vierteljahrshefte für Zeitgeschichte, 67(3), 443-453.

Przyborski, A., \& Wohlrab-Sahr, M. (2010). Qualitative Sozialforschung. Ein Arbeitsbuch. München: Oldenbourg.

Putnam, Robert D. (2016). Our Kids: The American Dream in Crisis. New York: Simon \& Schuster.

Reckwitz, A. (2017). Die Gesellschaft der Singularitäten. Zum Strukturwandel der Moderne. Frankfurt a. M.: Suhrkamp.

Reckwitz, A. (2020). Kampf um das Bürgerliche. Der Spiegel, 8. https://magazin.spiegel.de/SP/2020/8/ 169470965/index.html. Zugegriffen: 30. Apr. 2020.

Rehberg, K.-S. (Hrsg.). (2016). PEGIDA. Rechtspopulismus zwischen Fremdenangst und Wende-Enttäuschung? Analysen im Überblick. Bielefeld: transcript.

Reinders, H. (2016). Politische Sozialisation Jugendlicher. Entwicklungsprozesse und Handlungsfelder. In A. Gürlevik, K. Hurrelmann \& C. Palentien (Hrsg.), Jugend und Politik. Politische Bildung und Beteiligung von Jugendlichen (S. 85-101). Wiesbaden: Springer VS.

Reuband, K.-H. (2017). PEGIDA, Sachsen und die Fremdenfeindlichkeit. Warum es komplexerer und regionalspezifischer Analysen bedarf. Forschungsjournal Soziale Bewegungen, 30(1), 101-107.

Rohe, K. (1990). Politische Kultur und ihre Analyse. Probleme und Perspektiven der politischen Kulturforschung. Historische Zeitschrift, 250(132), 321-346.

Rohe, K. (1994). Politik. Begriffe und Wirklichkeiten. Eine Einführung in das politische Denken. Stuttgart: Kohlhammer.

Roth, R., \& Rucht, D. (2000). Jugendliche heute. Hoffnungsträger im Zukunftsloch? In R. Roth \& D. Rucht (Hrsg.), Jugendkulturen, Politik und Protest. Vom Widerstand zum Kommerz (S. 9-34). Opladen: Leske + Budrich.

Schäfers, B., \& Scherr, A. (2005). Jugendsoziologie. Einführung in Grundlagen und Theorien. Wiesbaden: VS.

Schenke, J., Finkbeiner, F., \& Neumann, A. (2020). Das Potenzial der Cleavage-Perspektive. Wahlverhalten als Oberflächenphänomen der politischen Kultur. Demokratie-Dialog, 6, 2-11. 
Schenke, J., Schmitz, C., Marg, S., \& Trittel, K. (2018). PEGIDA-Effekte? Jugend zwischen Polarisierung und politischer Unberührtheit. Bielefeld: transcript.

Schneekloth, U., \& Albert, M. (2019). Jugend 2019: Jugend und Politik: Demokratieverständnis und politisches Interesse im Spannungsfeld von Vielfalt, Toleranz und Populismus. In M. Albert, K. Hurrelmann \& G. Quenzel (Hrsg.), 18. Shell Jugendstudie - Eine Generation meldet sich zu Wort (S. 47-103). Weinheim: Beltz.

Schröder, M. (2018). Der Generationenmythos. KZfSS Kölner Zeitschrift für Soziologie und Sozialpsychologie, 70(4), 469-494.

Sellner, M. (2017). Identitär! Geschichte eines Aufbruchs. Schnellroda: Antaios.

Sommer, M., Rucht, D., Haunss, S., \& Zajak, S. (2019). Fridays for Future. Profil, Entstehung und Perspektiven der Protestbewegung in Deutschland (ipb working paper 2/2019).

Steinhaus, M., Heim, T., \& Weber, A. (2017). „So geht sächsisch!“. Pegida und die Paradoxien der ,sächsischen Demokratie‘. In T. Heim (Hrsg.), Pegida als Spiegel und Projektionsfläche. Wechselwirkungen und Abgrenzungen zwischen Pegida, Politik, Medien, Zivilgesellschaft und Sozialwissenschaften (S. 143-196). Wiesbaden: Springer VS.

Strübing, J., Hirschauer, S., Ayaß, R., Krähnke, U., \& Scheffer, T. (2018). Gütekriterien qualitativer Sozialforschung. Ein Diskussionsanstoß. Zeitschrift für Soziologie, 47(2), 83-100.

Vance, J. D. (2017). Hillbilly elegy. A memoir of a family and culture in crisis. New York: Harper Collins.

Vetter, A. (2006). Jugend: Ein Konzept und seine Messung. In E. Roller, F. Brettschneider \& J. W. van Deth (Hrsg.), Jugend und Politik: „Voll normal! “ (S. 25-53). Wiesbaden: VS.

Vorländer, H. (2016). PEGIDA. Entwicklung, Zusammensetzung und Deutung einer Empörungsbewegung. Wiesbaden: Springer VS.

Wagner, T. (2017). Die Angstmacher. 1968 und die Neuen Rechten. Berlin: Aufbau.

Walter, F. (2018). Zeiten des Umbruchs? Analysen zur Politik. Stuttgart: ibidem.

Weiß, V. (2017). Die autoritäre Revolte. Die Neue Rechte und der Untergang des Abendlandes. Stuttgart: Klett-Cotta. 